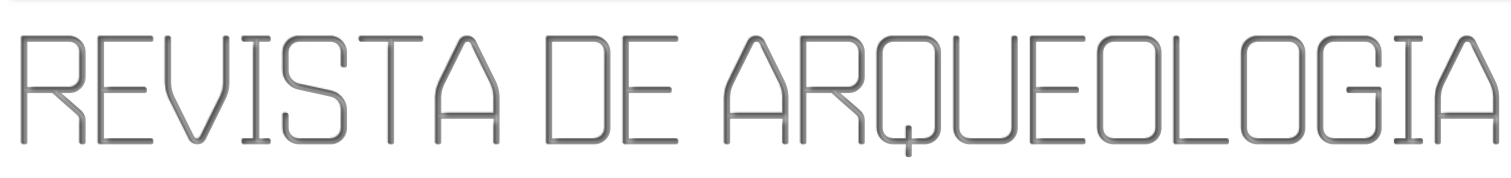

Volume 32 No. $22019 \quad$ Edição Especial: Museu Nacional [Volume 1]

\title{
RESGATANDO A MEMÓRIA DO ACERVO DOCUMENTAL DE PADBERG- DRENKPOL SOBRE O SÍTIO ARQUEOLÓGICO LAPA MORTUÁRIA DE CONFINS [REGIÃO DE LAGOA SANTA - MG]
}

Valéria Tavares ${ }^{*}$, Silvia Reis ${ }^{* \star}$, Matheus Monteiro ${ }^{\star * *}$, Claudia Rodrigues-Carvalho ${ }^{* \star * *}$

\section{RESUMO}

Inspirado pelas pesquisas em Lagoa Santa conduzidas pelo paleontólogo Peter Lund (século XIX), J. H. A. Padberg-Drenkpol realizou campanhas na região em 1926 e 1929. O principal sítio arqueológico trabalhado por ele foi a Lapa Mortuária de Confins (Confins, MG). Todo o acervo e informações do sítio ficaram sob a guarda do Museu Nacional - UFRJ. O incêndio de 2 de setembro de 2018 impactou profundamente pesquisas em curso, dentre as quais o projeto de caracterização da Lapa Mortuária de Confins. O objetivo deste artigo é resgatar a memória sobre o acervo documental deste sítio. Dessa forma, observou-se como o sítio pode ser caracterizado e contextualizado a partir dos dados das cartas e relatórios produzidos por Padberg-Drenkpol em suas expedições.

Palavras-chave: Lagoa Santa; Padberg-Drenkpol; Lapa Mortuária de Confins.

\footnotetext{
* Pesquisadora colaboradora, Departamento de Antropologia, Museu Nacional, UFRJ

E-mail: valeria_marquesds@hotmail.com. ORCID: https://orcid.org/0000-0001-5131-1712.

** Arqueóloga, Departamento de Antropologia, Museu Nacional, UFRJ E-mail: sreis@mn.ufrj.br. ORCID: https://orcid.org/0000-0003-2965-7834.

*** Bolsista PIBIC/UFRJ, Departamento de Antropologia, Museu Nacional, UFRJ.

E-mail: matheusmount.f@gmail.com. ORCID: https://orcid.org/0000-0002-6680-8606.

**** Professora Adjunta, PPGArq, Departamento de Antropologia, Museu Nacional, UFRJ.

E-mail: claudia@mn.ufrj.br. ORCID: https://orcid.org/0000-0002-9491-0659
} 


\title{
RESCUING THE MEMORY OF PADBERG-DRENKPOL'S DOCUMENTAL
}

\section{ARCHIVE ABOUT THE ARCHAEOLOGICAL SITE LAPA MORTUÁRIA DE \\ CONFINS [REGION OF LAGOA SANTA - MG]}

\begin{abstract}
Inspired by the research in Lagoa Santa conducted by paleontologist Peter Lund (19th century), JH Augusto Padberg-Drenkpol carried out several campaigns in the region from 1926 onwards. The main archaeological site worked was the socalled Lapa Mortuária de Confins. All the collection and information was under the protection of the National Museum. The fire of September 2, 2018, had a profound impact on several ongoing research, including the Lapa Mortuária de Confins characterization project. Thus, we aim to rescue the memory of this site, its characterization and contextualization based on information from the letters and reports produced by Padberg-Drenkpol in his expeditions.
\end{abstract}

Keywords: Lagoa Santa; Padberg-Drenkpol; Lapa Mortuária de Confins.

\section{RESCATANDO LA MEMORIA DEL ACERVO DOCUMENTAL DE PADBERG- DRENKPOL SOBRE EL SITIO ARQUEOLÓGICO LAPA MORTUÁRIA DE CONFINS [REGIÓN DEL LAGOA SANTA - MG]}

\begin{abstract}
RESUMEN
Inspirado por la investigación en Lagoa Santa realizada por el paleontólogo Peter Lund (siglo XIX), J. H. A. Padberg-Drenkpol realizó campañas en la región en 1926 y 1929. El principal sitio arqueológico en el que trabajó fue Lapa Mortuária de Confins. Toda la colección e información del sitio fueron depositadas en el Museo Nacional. El incendio del 2 de septiembre de 2018 impactó varias investigaciones en curso, incluido el proyecto de caracterización de Lapa Mortuária de Confins. El propósito de este artículo es recuperar la memoria sobre el acervo documental del sitio. Por lo tanto, se observó cómo el sitio puede caracterizarse y contextualizarse a partir de los datos de cartas e informes, producidos por Padberg-Drenkpol en sus expediciones.
\end{abstract}

Palabras clave: Lagoa Santa; Padberg-Drenkpol; Lapa Mortuária de Confins. 


\section{INTRODUÇÃO}

A cultura material, seja artefato, documento, ou, ainda, o corpo humano, possui em si um sentido de memória relacionado a lugares e pessoas, localizado em um tempo e espaço, à construção da memória de certos indivíduos e grupos sociais. Essa memória é construída a partir da realização de uma cadeia de ações que estão ligadas à construção da cultura material ou quando a sequência biográfica do objeto é preservada e por inúmeras, vezes pode ser reconstruída (AYOUB, 2016).

Assim, se faz necessário o resgate da memória das coleções que faziam parte do acervo do Museu Nacional (UFRJ) e que foram profundamente impactadas pelo o incêndio no dia 2 de setembro de 2018. O Museu Nacional é um espaço de produção de conhecimento e interação com a sociedade, partícipe da construção da memória coletiva e histórica brasileira. Entretanto, com o incêndio, diversas pesquisas que estavam em curso foram interrompidas, dentre elas o projeto de caracterização e contextualização do acervo proveniente do sítio arqueológico Lapa Mortuária de Confins (Confins, MG). Este local pertence ao complexo arqueológico de Lagoa Santa, região que fica no estado de Minas Gerais, no sudeste do Brasil (Figura 1). Essa região passou a ser conhecida internacionalmente, a partir do século XIX, pelos achados arqueológicos do paleontólogo Peter Lund (1835-1844).

Figura 1 - Localização do sítio Lapa Mortuária de Confins.

Elaborado por José Eduardo Abrahão.

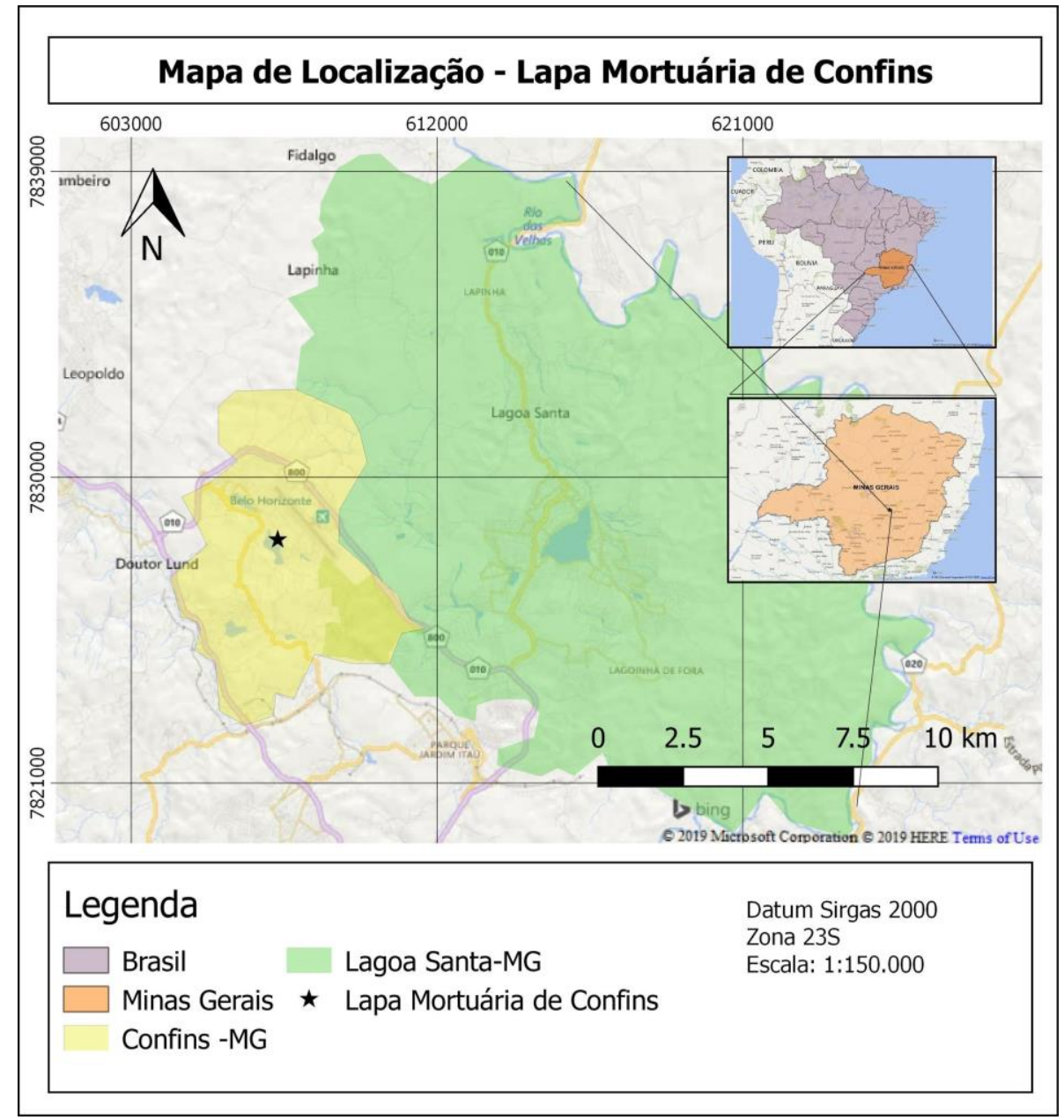


A região de Lagoa Santa possui diversas grutas, abrigos sob rochas e afloramentos calcários, que, devido às suas características, são favoráveis à preservação de vestígios ósseos. Dessa forma, a união entre as peculiaridades do solo e as cavidades naturais produz um efeito fossilizador, que garante a preservação do material arqueológico e paleontológico (AULER \& PILÓ, 2015 apud Da Glória et al, 2017). Segue abaixo a lista dos principais sítios arqueológicos da região de Lagoa Santa e municípios adjacentes (Tabela 1).

Tabela 1 - Sítios arqueológicos na região de Lagoa Santa e municípios adjacentes.

\begin{tabular}{ll} 
Município & Nome do sítio \\
\hline Matozinho & Abrigos de Cerca Grande \\
\hline Matozinho & Lapa D'Água \\
\hline Matozinho & Lapa da Moreira \\
\hline Matozinho & Lapa das Carrancas \\
\hline Matozinho & Lapa do Caetano \\
\hline Matozinho & Lapa das Boleiras \\
\hline Matozinho & Lapa do Santo \\
\hline Confins & Lapa Mortuária de Confins \\
\hline Lagoa Santa & Lapinha \\
\hline Pedro Leopoldo & Lapa Vermelha IV \\
\hline Pedro Leopoldo & Gruta do Sumidouro \\
\hline Pedro Leopoldo & Lapa do Eucalipto \\
\hline Pedro Leopoldo & Lapa Lagoa Funda \\
\hline Pedro Leopoldo & Lapa Mãe Rosa \\
\hline Pedro Leopoldo & Lapa da Limeira \\
\hline Pedro Leopoldo & Lapa do Acácio \\
\hline Pedro Leopoldo & Samambaia
\end{tabular}

Segundo Pedro da Glória (2012), a coleção de ossos humanos da região de Lagoa Santa é composta por 195 indivíduos datados entre 10.000 e 7.000 anos AP, distribuídos em três instituições brasileiras: Museu Nacional - UFRJ, Museu de História Natural da UFMG e Universidade de São Paulo. A caracterização da população pretérita de Lagoa Santa perpassa desde estudos craniométricos a análises de indícios sobre saúde e estilo de vida (MELLO E ALVIM, 1977; NEVES \& ATUI, 2004; MENDONÇA DE SOUZA et al., 2006; DA GLORIA, 2012; GONÇALVES et al., 2012).

Dos sítios arqueológicos da região de Lagoa Santa com material que fazia parte do acervo do Museu Nacional, destaca-se a chamada Lapa Mortuária de Confins (CNSA MG00321 e MGBF46). Registrada como uma pequena gruta no maciço da lagoa de Confins que se desenvolve por cerca de 25 metros no sentido Leste-Oeste, próximo à área urbana do distrito de Confins, está localizada em uma região de planalto, com compartimento topográfico de meia encosta, a 100 metros da lagoa. Foi considerada um sítio em uma gruta do período pré-colonial, com vestígios em superfície e em profundidade, com estruturas funerárias e arte rupestre definida como gravura (IPHAN, 1997).

Escavada por J. H. A. Padberg-Drenkpol (1877-1948), do Museu Nacional, a Lapa Mortuária de Confins é fundamental para a compreensão dos processos de povoamento, migração e circulação na região e no continente. Com no mínimo 80 indivíduos 
encontrados na expedição feita pelo Museu Nacional, esse sítio chama a atenção pela densidade de sepultamentos, levantando questões sobre o período de utilização e sua caracterização como espaço funerário. Todavia o material presente no acervo tinha sido pouco estudado, por problemas de contextualização, falta de clareza sobre estratigrafia, distribuição dos sepultamentos, além da falta de individualização. Apenas alguns crânios com maior grau de integridade permitiam vislumbrar elementos populacionais, ainda que de forma limitada.

Desde 2013, buscamos analisar os elementos possíveis de contextualização, cartas, manuscritos e fotos, em diálogo com o material osteológico humano presente no acervo. O projeto se encontrava na etapa de revisão completa do inventário de remanescentes humanos e identificação de amostras para datação, quando aconteceu o incêndio de 2 de setembro de 2018. Apenas parte do acervo de remanescentes humanos foi completamente inventariada. Os documentos produzidos por Padberg-Drenkpol, sob a guarda do Arquivo de Antropologia Física (Museu Nacional - UFRJ), tinham sido fotografados com êxito. No entanto os negativos de vidro não tinham sido ainda acessados, uma vez que necessitavam de restauração.

Destarte, faz-se premente resgatar a memória da pesquisa sobre do acervo documental e arqueológico do sítio após o incêndio. Buscamos entender como era o acervo documental e em que medida possibilita a caracterização da Lapa Mortuária de Confins. Para tanto, foram analisados os documentos previamente digitalizados.

\section{BREVE HISTÓRICO DAS PESQUISAS REALIZADAS EM LAGOA SANTA}

Lund realizou pesquisas na região de Lagoa Santa entre 1835-1844 (LUND, 1839; 1840; 1844; 1845). Visitou 800 grutas, coletando ossos humanos em apenas seis delas (Escrivania II e III, Baú, Braga, Vermelha e Sumidouro), sendo a maioria localizada nos maciços calcários do município de Pedro Leopoldo (MELLO E ALVIM, 1977; BELTRÃO, 2000). Após a descoberta da Gruta do Sumidouro, onde Lund identificou mais de 30 indivíduos enterrados com animais extintos, Lagoa Santa entrou no mapa internacional, uma vez que a maior parte do material foi levado para a Europa.

Posteriormente, em 1926 e 1929, as pesquisas em Lagoa Santa são retomadas pelo Museu Nacional através do pesquisador J.H.A. Padberg-Drenkpol. O objetivo dele era testar as ideias de Lund em novas escavações nas grutas de Lagoa Santa. Em sua segunda expedição, em 1929, na Lagoa Santa, Padberg encontrou a Lapa Mortuária de Confins com uma grande quantidade de vestígios ósseos humanos. Em 1937, foi realizada pelo Museu Nacional uma expedição em Lagoa Santa através dos pesquisadores Rui de Lima e Silva, Ney Vidal e Bastos de Ávila, na Lapa das Carrancas mais uma intervenção arqueológica (MELLO E ALVIM, 1977).

De 1930 a 1960, a Academia de Ciências de Minas Gerais também realizou na região pesquisas arqueológicas e paleontológicas. Os esqueletos humanos obtidos a partir dessas pesquisas foram analisados na década de 1990 no Laboratório de Estudos Evolutivos e Ecológicos Humanos da Universidade de São Paulo (LEEEH-USP) e mais tarde enviados para o Museu de História Natural e Jardim Botânico da Universidade Federal de Minas Gerais (MHNJB-UFMG) (MELLO E ALVIM, 1977; DA GLÓRIA et al, 2017).

Na ocasião, a Lapa Mortuária de Confins também foi pesquisada pela Academia de Ciências de Minas Gerais em 1935, e H. Walter concluiu que o ser humano era contemporâneo à megafauna. Ele chegou a essa conclusão por encontrar um crânio no mesmo lugar e nível onde foram encontrados vestígios fósseis. Mais tarde, os métodos de datação realizados por ele não foram considerados (MELLO E ALVIM, 1977).

Na década de 1950, Wesley Hurt da Universidade de Dakota do Sul junto com os pesquisadores brasileiros C. Faria, P. Couto, O. Blasi e F. Altenfelder tinham como 
objetivo principal estudar a contemporaneidade entre a megafauna extinta e os humanos a partir dos estudos de Lund. Realizaram pesquisas na Lapa das Boleiras e em Cerca Grande, encontrando 24 sepultamentos e grande quantidade de material lítico. Não foi encontrada megafauna associada ao material arqueológico, e a primeira datação radiocarbônica da região foi feita nesse momento, com datações de $9.028 \pm 120 \mathrm{AP}$ e $9.720 \pm 128$ AP (MELLO E ALVIM, 1977; DA GLÓRIA et al, 2017).

A segunda missão internacional feita em Lagoa Santa ocorreu de 1971-1975: a Missão Francesa liderada por A. Laming-Emperaire e com participação de A. Prous, M. Beltrão, A. Pires, S. Cunha, L. Pallestrini, A. Morães, M. J. Menezes e M. Andreatta. Na ocasião, a missão tinha como objetivo obter mais dados sobre a região, inventariar sítios e descobrir grutas intactas para um melhor estudo estratigráfico. Foi nessa campanha que a Lapa Vermelha IV foi escavada pela primeira vez, onde foi encontrado o esqueleto humano chamado de Luzia, com a crânio bem preservado e datado em torno de $11 \mathrm{mil}$ anos $\mathrm{AP}$, sendo considerado um dos esqueletos mais antigos das américas (MELLO E ALVIM, 1977; DA GLÓRIA et al, 2017).

Entre os anos de 1976 e 1979, André Prous, da Universidade Federal de Minas Gerais, escavou o sítio Grande Abrigo de Santana do Riacho, encontrando uma grande quantidade de material arqueológico relacionado a pinturas rupestres e 40 indivíduos do período do Holoceno, cuja curadoria foi feita pelo LEEEH-USP. Em 2000, Walter Neves e sua equipe realizaram em Lagoa Santa o projeto "Origens e microevolução do Homem na América: uma abordagem Paleoantropológica”. Tal projeto teve como objetivo levar para a região uma equipe de profissionais brasileiros que analisassem: ocupações pleistocênicas, origem biológica dos primeiros americanos, resiliência de forrageadores neotropicais, subsistência, cultura material, mobilidade e mudança social, formação de sítio, tafonomia, paleoclima e paleoambiente (DA GLÓRIA et al, 2017).

Atualmente as pesquisas relacionadas à Lagoa Santa estão ligadas aos estudos sobre a história populacional, e modelos de imigração dos primeiros americanos, rotas de migração e dispersão da população brasileira e americana vêm sendo feitos a partir de abordagens estatística moderna e biologia evolutiva (BERNARDO \& NEVES, 2016; HUBBE et al., 2015; BERNARDO, 2007; HUBBE et al., 2010, 2014). O entendimento sobre os padrões mortuários de Lagoa Santa mudaram a partir dos resultados do projeto Origens (NEVES et al., 2002; STRAUSS et al., 2015; 2016a, 2016b) e sobre a saúde dos primeiros habitantes da região: atividade física, estresse não específico durante, $\mathrm{o}$ crescimento, infecções, subsistência e violência (DA GLÓRIA, 2012).

\section{O ACERVO DOCUMENTAL DE PADBERG-DRENKPOL SOBRE A LAPA MORTUÁRIA DE CONFINS NO MUSEU NACIONAL}

O Arquivo de Antropologia Física do Museu Nacional possuía sob sua guarda diversos documentos, como cartas, relatórios (alguns ainda na versão rascunho), materiais oficiais e de divulgação (como alguns cartazes antigos de exposições), listas de coleções, além do primeiro livro de Tombo original do Setor de Antropologia Biológica (SANTOS \& SILVA, 2006). Desses, sete conjuntos documentais contêm materiais produzidos por Padberg-Drenkpol, dos quais três conjuntos versam diretamente sobre suas excursões e a Lapa Mortuária de Confins (Tabela 2). 
Tabela 2 - Conjuntos documentais com materiais produzidos por Padberg-Drenkpol, sob a guarda do Arquivo de Antropologia Física, Museu Nacional (SANTOS \& SILVA, 2006).

Registro no Arquivo de Antropologia Física AF.T.1.1.003
Descrição

(SANTOS \& SILVA, 2006)

"Correspondência e relatórios das excursões realizadas por J.A. PadbergDrenkpol, do Museu Nacional, na região de Lagoa Santa nos anos de 1926 e 1929, com o objetivo de recolher materiais fósseis e traçar um mapa da região, utilizando-se dos caminhos percorridos por Lund em suas expedições espeleológicas entre 1835 e 1844. Inclui rascunhos com cálculos. - S.l., de 12 out. 1926 a 1929. 4d., 42f."

\begin{tabular}{|c|c|}
\hline AF.T.1.1.007 & $\begin{array}{l}\text { "Lund e o Museu Nacional"”, sinopse das expedições e viagens realizadas } \\
\text { na região de Minas Gerais, desde 1834, escrita por Padberg-Drenkpol. - } \\
\text { S.l., } 6 \text { jun. 1931. 3d., 15f." }\end{array}$ \\
\hline AF.T.1.1.008 & $\begin{array}{l}\text { "Texto de Padberg-Drenkpol sobre Lagoa Santa, sua geologia e } \\
\text { paleontologia, para a ocasião das conferências da Escola Politécnica em } \\
\text { 1931. - S.l., [1931]. 1d., 1f." }\end{array}$ \\
\hline AF.T.1.1.016 & $\begin{array}{l}\text { "Notas para um relatório dos trabalhos de J.A. Padberg-Drenkpol em } \\
\text { 1937”, contendo pesquisas sobre o Homem de Confins, a raça de Lagoa } \\
\text { Santa e outros trabalhos realizados por ele. - S.l., jan. 1938. 1d., 3f." }\end{array}$ \\
\hline AF.T.1.1.017 & $\begin{array}{l}\text { "Correspondência trocada entre Arnaldo Cathoud, secretário geral da } \\
\text { Academia de Ciências de Minas Gerais, Heloísa Alberto Torres, Diretora } \\
\text { do Museu Nacional, Padberg-Drenkpol e Bastos de Ávila, a respeito do } \\
\text { trabalho publicado pelo argentino Antonio Serrano intitulado } \\
\text { "Concheros del Brasil", e explicações sobre os estudos do Museu Nacional } \\
\text { sobre o Homem de Confins. (...). - Rio de Janeiro, Belo Horizonte, de } 15 \\
\text { mar. a } 5 \text { jun. } 1939.5 \text { d., } 24 \mathrm{f} . "\end{array}$ \\
\hline AF.T.1.1.025 & $\begin{array}{l}\text { "Sinopse das viagens espeleológicas de Lund e das cavernas mais } \\
\text { importantes então visitadas, extraída pela maior parte dos seus diários', } \\
\text { texto traduzido por Padberg-Drenkpol a partir da biografia de Lund } \\
\text { elaborada por J. Reinhardt; e mapa manuscrito da região de Lagoa Santa, } \\
\text { provavelmente produzido por Padberg-Drenkpol. - S.l., s.d. 5d., 68f." }\end{array}$ \\
\hline
\end{tabular}

Os conjuntos documentais acima listados foram todos fotografados durante a execução da primeira etapa da pesquisa. Contendo rascunhos, anotações e versões corrigidas e datilografadas, esses conjuntos possibilitam também vislumbrar um pouco a vida de gabinete na época de Padberg-Drenkpol. Parte do material indica os estudos realizados e preparação para apresentações e falas públicas, além da necessária prestação de contas frente a instituição.

Em relatórios destinados a Roquette Pinto, Padberg-Drenkpol afirma que, desde seu ingresso no corpo científico da instituição, havia proposto sua ida à Lagoa Santa para continuar as pesquisas realizadas em 1835 e 1844 por Lund. Tinha como objetivo levar para o Museu Nacional os vestígios arqueológicos da região, uma vez que quase todo o material encontrado por Lund foi para o Museu de História Natural de Copenhagen, enquanto, no Brasil, permaneceu apenas um crânio, sob guarda do Instituto Histórico e Geográfico Brasileiro (PADBERG-DRENKPOL, 1926).

Para Padberg-Drenkpol, voltar à Lagoa Santa tinha a dupla função de conservar o que fora feito por Lund e desenvolver novas pesquisas na região. Havia a necessidade de reconstruir o quanto possível a obra de Lund, identificando as lapas por ele percorridas, organizando-as em mapas, apresentando o conjunto dos vestígios encontrados e discutindo sua relevância científica (PADBERG-DRENKPOL, 1926).

Apesar do objetivo central de Padberg-Drenkpol em seguir e aprofundar os estudos iniciados por Lund ao conduzir as campanhas na região de Lagoa Santa, tendo escavado sítios arqueológicos que interpretou como importantes em sua época, pouco publicou oficialmente. A maior parte do material textual produzido se resume a cartas, relatórios manuscritos e documentos datilografados. As publicações científicas de sua autoria 
encontradas no Boletim do Museu Nacional versam sobre diversos temas não relacionados à Lagoa Santa, como os títulos: "Situação histórico-cultural dos Karayás" (1926), "Carlos Teschauer" (1930) e "Mysteriosas galerias subterrâneas em Santa Catharina” (1933). Demais publicações em revistas, como o Boletim do Centro de Estudos Históricos (1937), ou livros sobre Herluf Winge (1927) ou um parecer sobre o acordo ortográfico (1948), mostram um lado público de Padberg que parece destoar de seus trabalhos em Lagoa Santa.

Todavia, em uma perspectiva antropológica da ciência, das idas e vindas da vida profissional, nota-se publicações consonantes com suas intenções dentro da instituição, como a publicação na Revista da Universidade do Rio de Janeiro (1932) intitulada "Estratigrafia e Paleontologia, com especial aplicação à Geologia do Brasil e à evolução dos organismos", uma das bases para pleitear a cadeira de Estratigrafia e Sedimentologia no Museu Nacional (KEULLER, 2017). Publicado na seção de Institutos não Universitários nessa mesma edição, encontra-se outros trabalhos sobre conteúdo de cursos ministrados como o "Curso especializado de antropometria" de Bastos d'Ávila. Da mesma forma, talvez o episódio que indica seu impacto como referência entre seus contemporâneos seja a participação no debate sobre a caracterização do chamado Homem de Confins (GASPAR NETO \& RODRIGUES-CARVALHO, 2017). Mesmo assim, sua contribuição para os estudos do povoamento do continente americano e da região de Lagoa Santa permanece como um marco na arqueologia brasileira.

\section{TEXTO, CONTEXTO E ACERVO}

Padberg-Drenkpol (1926) concluiu que era necessário continuar as pesquisas feitas por Lund, para a coleta de novos vestígios relacionados ao estudo da fauna antiga brasileira e uma maior compreensão quanto às origens humana nas Américas. Assim, o principal objetivo era esclarecer, a partir de novos achados e observações, se havia associação do ser humano com a fauna extinta em Lagoa Santa. Assim, Padberg realizou três excursões para Lagoa Santa: duas em 1926 e uma em 1929 (MELLO E ALVIM, 1977).

Ele relata que realizou duas expedições em 1926: de 17 de junho a 29 de julho e de 18 de setembro a 8 de dezembro. Foi na segunda excursão, no dia 7 de outubro de 1926, que encontrou o sítio arqueológico Lapa Mortuária de Confins. Descreveu que, ao percorrer Dr. Lund, antes de Confins, aproximadamente $3 \mathrm{~km}$ a nordeste da estação, encontrou a Lapa Vargem de Baixo às margens de uma lagoa (Figura 2). Esta seria a mais abundante em vestígios pré-históricos e paleontológicos, e esse motivo passou a denominá-la como Lapa Mortuária (PADBERG-DRENKPOL, 1926).

Padberg-Drenkpol afirma que:

Achando nessa lapa, que batizei de Lapa Mortuária, relíquias de uns 80 indivíduos humanos e muitos restos, especialmente dentes de animais extintos, mormente grandes, como de mastodonte, cavalos indígenas, Macharuchenia etc., a escavação foi feita sistematicamente, com auxílio de mais de meia dúzia de operários, durante todo o mês de outubro, acompanhando tudo com apontamentos, desenhos, photographias etc. Verificou-se que os restos humanos (entre os quais um ou outro esqueleto quase completo: os primeiros conhecidos da 'raça de Lagoa Santa'! - meia dúzia de crânios bem mensuráveis, mais de 50 mandíbulas, inúmeros dentes e especialmente muitos 'rochedos', i.e., as partes petrosas do osso temporal, muitas vezes a única testemunha dum indivíduo, estavam sem ordem, às vezes debaixo de grandes lajes ou blocos calcáreos (PADBERG-DRENKPOL, 1926). 
Figura 2 - Desenho com as localizações relativas de Confins, Lapa da Vargem de Baixo e demais locais relatados por Padberg-Drenkpol, acervo do Arquivo de Antropologia Física do Museu Nacional (PADBERG-DRENKPOL, 1926).

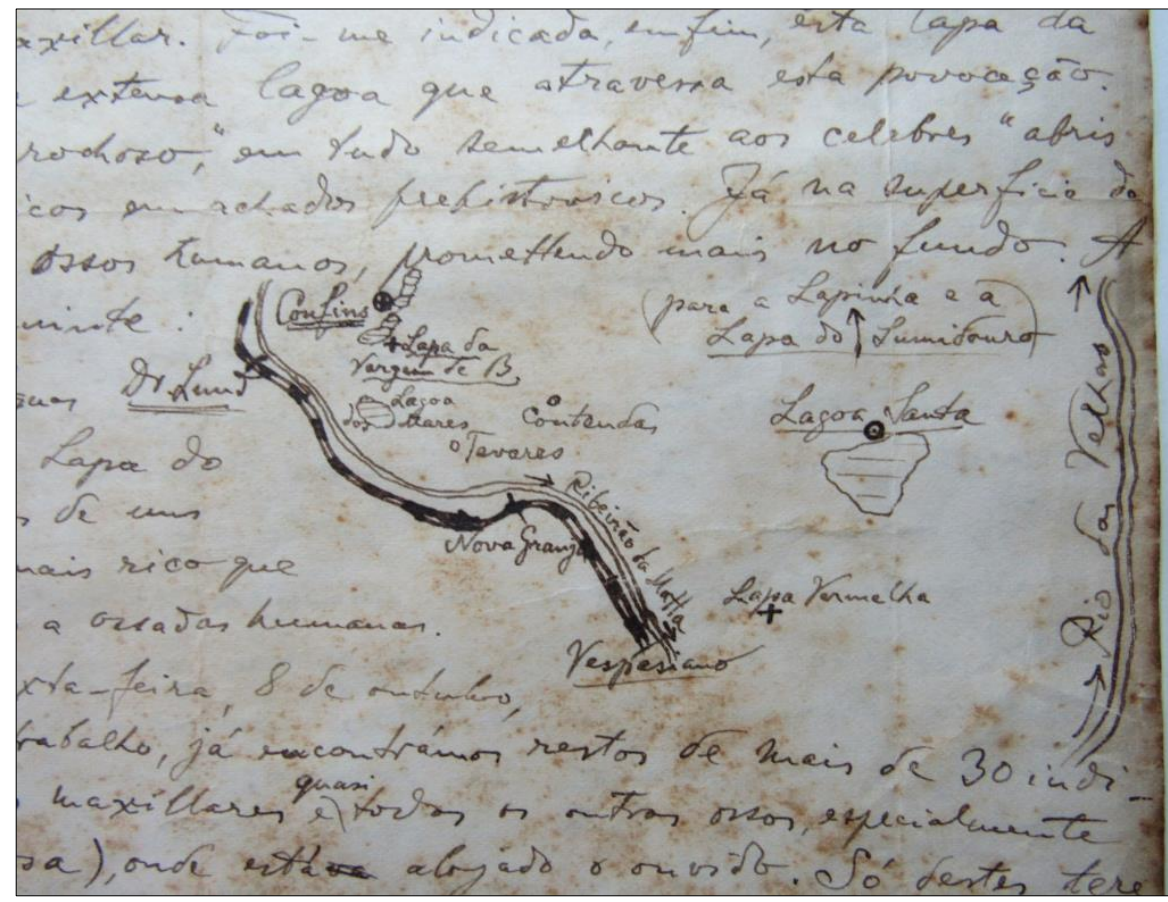

De acordo com a descrição do sítio, o solo estava muito perturbado, com alta dispersão de materiais, embaixo de lajes ou blocos de calcário, sedimento fino, com coloração preto-acinzentado e profundidade máxima de três metros. Havia maior quantidade de material arqueológico na entrada da lapa, onde tinha um abrigo sob rocha. No interior da gruta, foi percebido menos material arqueológico e mais vestígios de fauna do período do quaternário, com sedimento argiloso fino de coloração avermelhada (PADBERG-DRENKPOL, 1926).

Como resultado das duas excursões, foram coletadas doze caixas de vestígios de ossos humanos e de fauna, todos enviados ao Museu Nacional e catalogadas sob os números 20922 a 21327 . As 65 fotografias em chapas de vidro, em sua maioria com dimensão 13 x $18 \mathrm{~cm}$, foram arquivadas. Dados com informações de valor pré-histórico, paleontológico, geográfico e geológico também foram arquivados na instituição (PADBERG-DRENKPOL, 1926).

Padberg-Drenkpol (1926) se surpreendeu com a quantidade de remanescentes humanos do sítio. De acordo com ele, o sítio possuía covas médias e rasas e a escavação consistiu em extrair todo o aterro, que possuía sedimento fino e grandes rochas soltas, o que dificultou o trabalho. Este aterro era formado por entulhos de blocos de calcário vindo do teto que de certa forma ajudaram a preservar o interior da gruta.

No relatório o pesquisador afirma que verificou que as águas da lagoa próximo à gruta já havia há milhares de anos alcançado o sítio, e, no momento de sua visita, a gruta estava à 14 metros acima do nível da lagoa. A elevação das águas foi responsável por deixar na gruta vestígios de caramujos, ossinhos de peixe, cágados, jacarés, entre outros. Ao retirar o aterro da gruta, percebeu que havia uma fenda na parte interna que conduzia a um espaço de aproximadamente 20 metros de comprimento. Neste local já havia remanescentes humanos na superfície. Juntando esse espaço de 20 metros com o interior da gruta, a área com vestígios de ossos humanos correspondeu à 100 metros quadrados (PADBERG-DRENKPOL, 1926). Os enterramentos não possuíam uma ordem aparente, 
e os vestígios estavam mal preservados. Padberg não cita nas cartas a presença de pinturas rupestres na Lapa Mortuária de Confins, mas, na descrição dada pelo IPHAN, como visto acima, afirma a presença de pinturas rupestres na gruta.

Além de ossos humanos e fauna, foram encontrados instrumentos líticos utilizados para caça, facas retocadas, machados geralmente de seixos rolados e polidos no gume e outros trabalhados, ferramentas de polir e socar, e fragmentos de cerâmica. Segundo Padberg-Drenkpol, o material faunístico encontrado junto com os vestígios humanos era de animais recentes (veados, porcos do mato) e não megafauna (PADBERG-DRENKPOL, 1926).

Assim, mesmo diante dos limites da produção bibliográfica de Padberg-Drenkpol, a partir dos textos manuscritos e datilografados, a Lapa Mortuária de Confins foi caracterizada como um abrigo sob rocha, próximo a uma lagoa. Sua entrada estava bloqueada por um desmoronamento do teto com rochas de calcário. Havia uma certa quantidade de remanescentes humanos na entrada da gruta em uma limpeza de superfície, entretanto em seu interior havia uma maior quantidade de material em uma profundidade de até 3 metros. Toda a área com remanescentes humanos correspondeu a 100 metros quadrados, e não havia uma ordem para os sepultamentos, segundo PadbergDrenkpol. Possíveis enchentes na caverna podem indicar a possibilidade de alteração do espaço.

Esta pesquisa foi motivada inicialmente frente ao que tinha sido denominado como "refugo", que se encontrava no laboratório sem tombamento, mas já tratado e com novo acondicionamento. Apenas pequenos fragmentos amarelados de jornal aderidos aos ossos sugeria como poderia ter sido o acondicionamento original. A análise detalhada desse conjunto suscitou o aprofundamento da caracterização da coleção da Lapa Mortuária de Confins como um todo, frente à documentação depositada no Arquivo de Antropologia Física.

A descrição feita por Padberg-Drenkpol indica a princípio a possibilidade de dois espaços: entrada e interior da Lapa. Ao nos voltarmos para a coleção de remanescentes humanos, nota-se a falta de registro distinguindo a localização no sítio, sem dados como nível ou profundidade, ou ainda se proveniente da entrada ou do interior da Lapa Mortuária de Confins (LIVRO DE TOMBO DO SETOR DE ANTROPOLOGIA BIOLÓGICA, s.d.). Assim, a partir de uma análise preliminar, foi possível pensar a hipótese de dois eventos ou momentos distintos de uso da Lapa Mortuária Confins, entendendo a coleção como composta por dois conjuntos de remanescentes humanos. Para testá-la, estava sendo realizada a revisão do inventário completo desta coleção, análise de tafonomia do material e seleção de amostras dos hipotéticos dois conjuntos de ossos.

\section{CONSIDERAÇÕES FINAIS}

O incêndio de 2 de setembro de 2018 na sede do Museu Nacional impactou profundamente em diversas pesquisas. Esta pesquisa se encontrava na etapa de revisão completa do inventário de remanescentes ósseos humanos, com o objetivo de identificação de amostras ósseas para datação. Considerando os resultados da contextualização da coleção frente às cartas e demais documentos de Padberg-Drenkpol, pretendíamos testar a hipótese se os dois tipos de uso do espaço na Lapa Mortuária de Confins correspondiam a dois momentos distintos de ocupação do espaço, próximos ou distantes. Da mesma forma, tínhamos o intuito de reorganizar a coleção, separando e documentando os dois conjuntos de remanescentes humanos.

No presente momento, buscamos registrar a memória da coleção da Lapa Mortuária de Confins e trajetória de pesquisas, com o objetivo de promover sua identificação e 
recuperação, na medida do possível. Somente após a conclusão das etapas de resgate e de inventário será possível avaliar e apresentar informações sobre a coleção de remanescentes humanos da Lapa Mortuária de Confins.

AGRADECIMENTOS

Agradecemos ao Prof. Dr. Ricardo Ventura Santos e a Mônica Costa dos Santos Coelho, pelo apoio e acesso ao Arquivo de Antropologia Física (Museu Nacional). Agradecemos também a todos que apoiam os esforços de resgate e reconstrução do Museu Nacional. Agradecemos ao arqueólogo José Eduardo Abrahão pela elaboração do mapa. 


\section{REFERÊNCIAS BIBLIOGRÁFICAS}

AYOUB, Munir Lutfe. 2016. Arqueologia da memória estudos e teorias para um novo rumo da compreensão dos artefatos. Revista Territórios es Fronteiras, Cuiabá, 9(2): 257-273.

BELTRÃO, Maria. 2000. Ensaio de Arqueologia. Rio de Janeiro, Zit Gráfica e Editora.

BERNARDO, Danilo Vincensotto. 2007. Afinidades morfológicas intra e extra-continentais dos paleoíndios de Lagoa Santa: uma nova abordagem.319 f. Dissertação (Mestrado em Biologia Genética) - Universidade de São Paulo, São Paulo.

BERNARDO, Danilo Vincensotto; NEVES, Walter Alves. 2016. Os esqueletos de Lagoa Santa e a morfologia craniana dos primeiros americanos. In: DA-GLORIA, Pedro; NEVES, Walter Alves; HUBBE, Mark (Org.). Lagoa Santa: história das pesquisas arqueológicas e paleontológicas. São Paulo: Editora Annablume, 249-274.

CUNHA, Fausto Luiz de Souza. 1975. O Patrimônio arqueológico das cavernas brasileiras. ANAIS do $10^{\circ}$ Congresso Nacional de Espeleologia Ouro Preto MG, 14-16 de novembro de 1975 Sociedade Brasileira de Espeleologia.

DA-GLÓRIA, Pedro. 2012. Health and lifestyle in the paleoamericans: early holocene biocultural adaptation at Lagoa Santa, Central Brazil. Columbus, The Ohio State University.

DA-GLÓRIA, Pedro; NEVES, Walter; HUBBE, Mark. 2016. História das Pesquisas em Lagoa Santa, Minas Gerais: ossos humanos e patrimônio arqueológico. IV Seminário de Preservação de Patrimônio Arqueológico. AT Rio de Janeiro, p. 433-443.

DA-GLÓRIA, Pedro. et al. 2017. História das pesquisas bioarqueológicas em Lagoa Santa, Minas Gerais, Brasil. Bol. Mus. Para. Emílio Goeldi. Cienc. Hum., Belém, 12(3): 919-936.

GASPAR NETO, Verlan Valle; RODRIGUES-CARVALHO, Claudia. 2017. The Physical Anthropology Archives of the National Museum of Rio de Janeiro: Lagoa Santa in the First Half of the Twentieth Century. In: Da-Gloria P., Neves W., Hubbe M. (eds) Archaeological and Paleontological Research in Lagoa Santa. Springer, Cham.

GONÇALVES, Assis; MAIO, Marcos Chor; SANTOS, Ricardo Ventura. 2012. Entre o laboratório de antropometria e a escola: a antropologia física de José Bastos de Ávila nas décadas de 1920 e 1930. Boletim do Museu Paraense Emílio Goeldi, 7(3):671-686.

HUBBE, Mark; NEVES, Walter Alves; HARVATI, Katerina. 2015. Testing evolutionary and dispersion scenarios for the settlement of the New World. PLoS ONE, 5(6): e11105.

HUBBE, Mark; OKUMURA, Mercedes; BERNARDO, Danilo V.; NEVES, Walter Alves. 2014. Cranial morphological diversity of Early, Middle, and Late Holocene Brazilian groups: implications for human dispersion in Brazil. American Journal of Physical Anthropology, New Jersey, 155(4): 546-558.

HUBBE, Mark; STRAUSS, André; HUBBE, Alex; NEVES, Walter Alves. 2015. Early South americans cranial morphological variation and the origin of American biological diversity. PLoS ONE, 10(10): e0138090.

HUBBE, Mark; NEVES, Walter Alves; HARVATI, Katerina. 2010. Testing evolutionary and dispersion scenarios for the settlement of the New World. PLoS ONE, San Francisco, v. 5, n. 6, p. e11105. https://doi.org/10.1371/journal.pone.0011105.

IPHAN. 1997. Cadastro Nacional de sítios arqueológicos. Lapa Mortuária de Confins (MG00321e $M G B F 46)$.

KEULLER, Adriana. T. A. M. 2017. The Anthropological Studies of Lagoa Santa in the National Museum of Rio de Janeiro: Insertion, Debates, and Scientific Controversies at the Turn of the Nineteenth to the Twentieth Century. In: Da-Gloria P., Neves W., Hubbe M. (eds) Archaeological and Paleontological Research in Lagoa Santa. Springer, Cham.

LIVRO de Tombo do setor de Antropologia Biológica, s.d 
LUND, Peter W. 1839. Coup-d'oeil sur les espèces éteintes de mammiferes du Brésil, extrait de quelques mémoires présentés à l'Académie Royale des Sciences de Copenhague. Ann. Sc. Nat. Paris (2ª série), Zoologie, 11:214-239.

LUND, Peter W. 1840. Nouvelles recherches sur la faune fossile du Brésil. Ann. SC. Nat. Paris (2a série), Zoologie, 13:310-319.

LUND, Peter W. 1844. Carta do Dr. Lund, escripta da Lagoa Santa (Minas Geraes) a 21 de abril de 1844. Revista Trimensal de História e Geografia do Inst. Hist. Geog. Brasileiro, Rio de Janeiro, 6:334-342 (2a edição em 1865).

LUND, Peter W. 1845. Remarques sur les ossements fossiles trouvés dans les cavernes du Brésil. Mém. Sc. R. Ant. Nord, Copenhague, (1845-1849), 49-77.

MELLO E ALVIM, Marília C. 1977. Os antigos habitantes da área arqueológica de Lagoa Santa (MG-Brasil) - Estudo Morfológico. Arquivos do Museu de História Natural da Universidade Federal de Minas Gerais, 2:119-174.

MENDONÇA DE SOUZA, Sheila; RODRIGUES-CARVALHO, Claudia; SILVA, Hilton; LOCKS, Martha. 2006. Revisitando a discussão sobre o quaternário de Lagoa Santa e o povoamento das Américas: 160 anos de debates científicos. In: SILVA, Hilton \& RODRIGUES-CARVALHO, Claudia (org.), Nossa origem: o povoamento das Américas. Rio de Janeiro, Vieira e Lent, pp.19-43.

NEVES, Walter; ATUI, João Paulo. 2004. O mito da homogeneidade biológica na população paleoíndia de Lagoa Santa: implicações antropológicas. Revista de Antropologia, 47(1):159-205.

NEVES, Walter Alves; HUBBE, Mark; ARAUJO, Astolfo G. M. 2002. A late-Paleoindian secondary ritual burial from Lagoa Santa, Minas Gerais, Brazil. Current Research in the Pleistocene, Texas, v.19, p. 83-85.

PADBERG-DRENKPOL, Jorge Henrique Augusto. 1926. Relatório de duas excursões à região calcária de Lagoa Santa em 1926. Manuscrito apresentado à Seção de Antropologia e Etnologia do Museu Nacional do Rio de Janeiro.

PADBERG-DRENKPOL, Jorge Henrique Augusto. 1929. Relatório de duas excursões à região calcária de Lagoa Santa em 1929. Manuscrito apresentado à Seção de Antropologia e Etnologia do Museu Nacional do Rio de Janeiro.

PADBERG-DRENKPOL, Jorge Henrique Augusto. 1926. Situação histórico-cultural dos Karayá. Boletim do Museu Nacional, 2(6): 71-82.

PADBERG-DRENKPOL, Jorge Henrique Augusto. 1927. Um benemérito do Brasil, o dinamarquês Herluf Winge, classificador dos achados paleontológicos de Lund (1857-1923). Rio de Janeiro, Museu Nacional.

PADBERG-DRENKPOL, Jorge Henrique Augusto. 1930. Carlos Teschauer. Boletim do Museu Nacional, 6(4):265-269.

PADBERG-DRENKPOL, Jorge Henrique Augusto. 1932. Estratigrafia e Paleontologia, com especial aplicação à Geologia do Brasil e à evolução dos organismos. Revista da Universidade do Rio de Janeiro, 2(2):249-260.

PADBERG-DRENKPOL, Jorge Henrique Augusto. 1933. Mysteriosas galerias subterrâneas em Santa Catharina. Boletim do Museu Nacional, 9(1):83-91.

PADBERG-DRENKPOL, Jorge Henrique Augusto. 1937. Recordações históricas do Rio através de velhas inscrições latinas: dois dísticos dos antigos jesuítas do Rio e um epitáfio memorável. Boletim do Centro de Estudos Históricos, 2(2):18-25.

PADBERG-DRENKPOL, Jorge Henrique Augusto. 1948. Parecer sobre o acordo ortográfico interacadémico da língua portuguesa, aprovado pela academia brasileira de filologia. Rio de Janeiro, J. do Commercio. 
PEREZ, Cassiana. 2009. Paleoecologia de mamiferos viventes como ferramenta na caracterização do ambiente holocênico de Lagoa Santa, MG. São Paulo, USP.

SANTOS, Ricardo Ventura; SILVA, Maria Celina. 2006. Inventário analítico do Arquivo de Antropologia Física do Museu Nacional. Série Livros 14, Rio de Janeiro, Museu Nacional.

STRAUSS, André M. 2016a. As práticas mortuárias na região de Lagoa Santa. In: DA-GLORIA, Pedro; NEVES, Walter Alves; HUBBE, Mark (Org.). Lagoa Santa: história das pesquisas arqueológicas e paleontológicas. São Paulo: Editora Annablume, p. 299-322.

STRAUSS, André. 2016b. Os padrões de sepultamento do sítio arqueológico Lapa do Santo (Holoceno Inicial, Brasil). Boletim do Museu Paraense Emílio Goeldi. Ciências Humanas, Belém, 11(1): 243-276.

STRAUSS, André; OLIVEIRA, Rodrigo Elias; BERNARDO, Danilo V.; SALAZAR-GARCÍA, Domingo C.; TALAMO, Sahra; JAOUEN, Klervia; HUBBE, Mark; BLACK, Sue; WILKINSON, Caroline; RICHARDS, Michael Phillip; ARAUJO, Astolfo G. M.; KIPNIS, Renato; NEVES, Walter Alves. 2015. The oldest case of decapitation in the New World (Lapa do Santo, East-Central Brazil). PLoS ONE, 10(9): e0137456. 